\title{
An extensions theory setting for scattering by breathing bag
}

\author{
Yu. A. Kuperin, K. A. Makarov, ${ }^{a}$ and B. S. Pavlov \\ Leningrad State University, Leningrad, Union of Soviet Socialist Republics
}

(Received 5 October 1988; accepted for publication 21 June 1989)

\begin{abstract}
A model of the scattering of a structureless pointlike particle on a spherical bag with an internal structure, imitating "quark" degrees of freedom, is considered. It is assumed that the bag is a dynamical object and its radius plays the role of additional dynamical variable. The energy of the collision is distributed among the quark excitations and the vibration degrees of freedom of the bag surface. In the frame of the theory of extensions the Hamiltonian of the coupled bag-quark system interacting with the pointlike particle is constructed. The formal multichannel $S$ matrix of the problem is obtained.
\end{abstract}

\section{INTRODUCTION}

Let us consider two conservative quantum systems $Q_{1}$ and $Q_{2}$ with Hamiltonians $H_{1}$ and $H_{2}$ acting in Hilbert spaces $\mathscr{H}_{1}$ and $\mathscr{H}_{2}$, respectively. We assume that the system $Q_{1}$ consists of two noninteracting subsystems $Q^{b}$ and $Q^{q}$. Let the Hamiltonian $H_{1}$ of the system $Q_{1}$ be a direct sum of the corresponding Hamiltonians $H^{b}$ and $H^{q}$, which determine independent evolutions of the subsystems: $H_{1}=H^{b} \oplus H^{q}$ in the Hilbert space $\mathscr{H}_{1}=\mathscr{H}^{b} \oplus \mathscr{H}^{a}$. The total Hamiltonian $H_{0}$ of the system $Q_{1} \cup Q_{2}$ is represented as a tensor sum of the operators $H_{i}, i=1,2$,

$$
H_{0}=H_{1} \otimes I_{2}+I_{1} \otimes H_{2} .
$$

Because of the decomposition $\mathscr{H}_{1}=\mathscr{H}^{b} \oplus \mathscr{H}^{q}$, the relation (1) can be written in the form

$$
\begin{aligned}
H_{0}= & \left(H^{b} \otimes I_{2}+I_{b} \otimes H_{2}\right) \\
& \oplus\left(H^{q} \otimes I_{2}+I_{q} \otimes H_{2}\right) \\
\equiv & L_{1} \oplus L_{2} .
\end{aligned}
$$

One could switch on an interaction between the subsystem in the composite quantum system $Q_{1} \cup Q_{2}$, as was done in Ref. 1 . The method of the paper ${ }^{1}$ deals with the situation in which the total Hilbert space is a direct sum of its subspaces. An interaction arises as a result of construction of a set of self-adjoint extensions of some symmetric operator. This symmetric operator is the result of the restriction of the direct sum of initial Hamiltonians $L_{1} \oplus L_{2}$. This set, in particular, contains the initial Hamiltonian of the composite system. Any other self-adjoint extension can be interpreted as the total Hamiltonian determining the coupled dynamics of the subsystems and the interaction between them.

In this paper we use the techniques of Refs. 1-4 for the operators $L_{i}, i=1,2$, of special structure (2), i.e., in the case when the total energy of the subsystems $Q^{b}, Q^{q}$, and $Q_{2}$ is the sum of the subsystem energies. We consider the scattering of a pointlike particle on a dynamical quark bag ${ }^{5}(\mathrm{DQB})$ as an illustration of the scheme described above.

In this interpretation the operator $H^{b}$ determines the dynamics of the DQB surface, $H^{q}$ corresponds to the internal (quark) degrees of freedom, and $\mathrm{H}_{2}$ is the operator of kinetic energy of the pointlike particle. We construct an interaction between the abovementioned degrees of freedom

\footnotetext{
a) Present address: Freie Universität Berlin, Berlin (West), Germany.
}

on the base of the extensions theory. ${ }^{1-4}$ It will be shown that this approach leads to a nontrivial scattering matrix.

\section{MODEL}

Let us assume that the dynamics of the bag surface is given by the self-adjoint operator acting in the Hilbert space $\mathscr{H}^{b}=L_{2}(0, \infty)^{5}$,

$$
H^{b} \chi=\left(-\frac{1}{2 M} \frac{d^{2}}{d R^{2}}+V(R)\right) \chi,
$$

with boundary condition

$$
\chi(0)=0 \text {. }
$$

To simulate the breathing character of the system one should choose the potential $V(R)$ with confinement: $V(R) \rightarrow \infty$ as $R \rightarrow \infty$.

In this case the Hamiltonian $H^{b}$ has the pure discrete spectrum $\sigma\left(H^{b}\right)=\left\{\epsilon_{n]}\right\}_{n=0}^{\infty}$ and its eigenfunctions form the basis in the Hilbert space $\mathscr{H}^{b}$.

The dynamics of quark degrees of freedom inside the DQB is determined by a self-adjoint operator $H^{q}$, acting in an abstract Hilbert space $\mathscr{H}^{b}$. The nature of the Hamiltonian $H^{b}$ can be treated in different ways. It might be understood as the few-body Schrödinger operator with confining potentials (if one needs a nonrelativistic description); or as some relativistic Hamiltonian. Since there is no adequate mathematical description of multiquark dynamics at distances of the order of the confinement, we use the information about the structure of the spectrum of the Hamiltonian $H^{q}$ only, omitting concrete details of the evolution of internal (quark) degrees of freedom. In this case the bound states of $H^{q}$ can be treated as quark excitations of the DQB.

Finally, the role of the operator $\mathrm{H}_{2}$ is played by the Hamiltonian of the free particle

$$
\begin{aligned}
& H_{2} u=-\frac{1}{2 m} \frac{d^{2}}{d r^{2}} u, \quad r \geqslant 0, \\
& u(0)=0,
\end{aligned}
$$

in the Hilbert space $L_{2}(0, \infty)$. Let us assume that the interaction between the particle and DQB switches on if and only if the coordinates $R$ and $r$ are equal, and the pointlike particle does not penetrate into the bag. As a consequence, the configuration space $\mathscr{R}_{+}^{2}$ is divided by the line $y=\alpha x$ into two sectors $V^{ \pm}=\{y \geqslant \leqslant \alpha x\}$, where $\alpha=(m / M)^{1 / 2}$, 
$x=\sqrt{2 M} R, y=\sqrt{2 m r}$, and the scattering should be considered in "physical" sector $V^{+}$only.

The condition of nonpenetrability into the bag leads to the following rather simple model of an interaction between the quantum systems $Q^{b}$ and $Q_{2}$. One needs to solve the Schrödinger equation in the physical sector $V^{+}$:

$$
\left(-\frac{\partial^{2}}{\partial x^{2}}-\frac{\partial^{2}}{\partial y^{2}}+V(x)-E\right) \psi(x, y)=0,
$$

with the boundary conditions

$$
\left.\psi(x, y)\right|_{y=\alpha x}=0, \quad \psi(x, 0)=0 .
$$

To include the interactions between the systems $Q^{b}, Q^{q}$, and $Q_{2}$ in accordance with Refs. $1-4$, let us restrict the domain of the operator $L_{1}$ to the set of all smooth functions in the sector $V^{+}$, which vanish at the vicinity of the line $y=\alpha x$. The symmetric operator $L_{10}$, restricted in such a way, has infinite deficiency indices. The boundary form ${ }^{1-4}$ of its adjoint operator $L_{10}^{*}$ can be calculated as follows:

$$
\left\langle L_{10}^{*} \psi, \varphi\right\rangle-\left\langle\psi, L_{10}^{*} \varphi\right\rangle=\int_{r}\left(\partial_{n} \psi \bar{\varphi}-\partial_{n} \bar{\varphi} \psi\right) d \gamma,
$$

where $\partial_{n}$ denotes the normal derivative of the functions $\psi, \varphi$ on the curve $\gamma$. [Of course, the expression for the boundary form (9) on the domain $\mathscr{D}\left(L_{10}^{*}\right)$ needs some regularization. But for further purposes it is sufficient to consider the boundary form (9) only on the class of smooth functions, which do not satisfy any boundary condition on the line $\gamma$. In this case one does not need any regularization and (9) holds in the ordinary sense.]

The next step of the scheme $e^{1-4}$ is the restriction of the operator $L_{2}$ to some symmetric operator $L_{20}$. Since the operator $L_{2}=\mathrm{H}_{q}+\mathrm{H}_{2}$ acts in the tensor product of the Hilbert spaces $\mathscr{H}^{q} \otimes \mathscr{H}_{2}$ we shall restrict the domain of the operator $H^{q}$ only, so that the fixed elements $\theta$ from $\mathscr{H}^{q}$ be the deficiency element for the restriction $H_{0}^{q}$,

$$
\mathscr{D}\left(H_{0}^{q}\right)=\left\{\left(H^{q}-i I\right)^{-1} \psi, \psi \epsilon \mathscr{H}^{q} \ominus \theta\right\} .
$$

We call the coefficients $\epsilon_{n}^{ \pm}$of the decomposition

$$
\begin{aligned}
u= & u_{0}+\epsilon_{n}^{+} H^{q}\left(H^{q}-i I\right)^{-1} \theta+\epsilon_{n}^{-}\left(H^{q}-i I\right) \theta, \\
& u_{0} \in \mathscr{D}\left(H_{0}^{q}\right), \quad u \in \mathscr{D}\left(H_{0}^{q^{*}}\right)
\end{aligned}
$$

of arbitrary element $u$ from the domain of the adjoint operator $H_{0}^{q^{*}}$, the boundary values. In a general case, the boundary values $\epsilon^{ \pm}$of the elements $u$ from $\mathscr{D}\left(L_{20}^{*}\right)$ become the functions of the variable $y$ and the boundary form of the operator $L_{20}^{*}$ is given by

$$
\begin{aligned}
\left\langle L_{20}^{*} u, v\right\rangle-\left\langle u, L_{20}^{*} v\right\rangle= & \int_{R^{+}}\left[\epsilon_{u}^{-}(y) \overline{\epsilon_{v}^{+}(y)}\right. \\
& \left.-\epsilon_{v}^{-}(y) \overline{\epsilon_{u}^{+}(y)}\right] d y,
\end{aligned}
$$

In order to construct a self-adjoint extension $H$ of the operator $L_{10} \oplus L_{20}$, in accordance with our general method, one should impose on the line $\gamma$ such boundary conditions that nullify the sum of the boundary forms (9) and (12). One of the simplest possibilities to do it is to study the following boundary conditions, mixing all the channels together:

$$
\left.\partial_{n} \psi\right|_{\gamma}=\beta \epsilon^{-}(y), \quad \epsilon^{+}(y)=\left.\beta \psi\right|_{\gamma}, \quad \beta \in R^{\prime} .
$$

\section{SCATTERING PROBLEM}

The spectral analysis of the total two-channel Schrödinger equation

$$
(H-E) \psi=0
$$

can be reduced to some boundary-value problem in the sector $V^{+}$. More precisely, it can be shown $n^{4}$ that the boundary values $\epsilon^{ \pm}$on the solutions of the problem (14) are connected with the relation

$$
\epsilon^{-}(y)=Q\left(E+\frac{d^{2}}{d y^{2}}\right) \epsilon^{+}(y) .
$$

Here $Q\left(E+d^{2} / d y^{2}\right)$ is the integral operator acting in the space $L^{2}\left(R_{+}^{1}\right)$ with the kernel $Q\left(y, y^{\prime}, z\right)$,

$$
Q\left(y, y^{\prime}, z\right)=\frac{1}{2 \pi} \oint_{\Gamma} \Delta(\zeta) r_{0}\left(y-y^{\prime}, z-\zeta\right) d \zeta,
$$

where $r_{0}(u)=\left(H_{2}-z\right)^{-1}$ is the resolvent of the operator $-d^{2} / d y^{2}$ with boundary conditions $\left.\psi\right|_{y=0}=0, \Delta(\zeta)$ is the Schwartz integral of the spectral measure of the operator $H^{q}$, and the counter $\Gamma$ encircles the spectrum of the Hamiltonian $H^{q}$ in the complex plane $(\xi)$.

Equality (15) reduces the problem (14) to the search of the components of the wave function $\Psi$ in the space $\mathscr{H}^{q} \otimes \mathscr{H}_{2}$ as the solutions of the following boundary value problem in the sector $V^{+}$:

$$
\left(-\frac{\partial^{2}}{d x^{2}}-\frac{\partial^{2}}{d y^{2}}+V(x)-E\right) \psi=0
$$

with energy-dependent boundary conditions

$$
\begin{aligned}
& \left.\partial_{n} \psi\right|_{\gamma}=\left.\beta^{2} Q\left(E+\frac{d^{2}}{d y^{2}}\right) \psi\right|_{\gamma}, \\
& \left.\psi\right|_{x=0}=0,
\end{aligned}
$$

and appropriate asymptotic conditions at infinity. All the information about internal degrees of freedom penetrates into the boundary-value problem (17)-(19) through the Schwartz integral $\Delta(\zeta)$ of the Hamiltonian $H^{q}$,

$$
\begin{aligned}
& \Delta(\zeta)=\int \frac{\lambda_{\zeta}+1}{\lambda-\zeta} d\left\langle E_{\lambda} \theta, \theta\right\rangle, \\
& H^{q}=\int \lambda d E_{\lambda}^{q} .
\end{aligned}
$$

Here $\theta \in \mathscr{H}^{q}$ is a vector parameter of the theory [see (10)]. The function $\Delta(\xi)$ is an analytical function with a positive imaginary part in the upper half-plane of the complex variable $\zeta$. If the Hamiltonian $H^{q}$ is a finite-dimensional selfadjoint operator with a simple spectrum, $\lambda_{s}$ are its eigenvalues and $E_{s}$ are the corresponding orthogonal eigenprojectors, then $\Delta(\zeta)$ is a rational function,

$$
\Delta(\zeta)=\sum_{s} \frac{\zeta \lambda_{s}+1}{\lambda_{s}-\zeta}\left\langle E_{s} \theta, \theta\right\rangle_{\mathscr{K}^{q}} .
$$

The formal solution ${ }^{6}$ of the problem (17)-(19) can be represented in the form

$$
\psi_{m}(x, y)=\sum_{n}\left\{\delta_{m n} e^{-i k_{n} y}-S_{m n} e^{i k_{n} y}\right\} \chi_{n}(x),
$$

where $k_{n}=\sqrt{E-\epsilon_{n}}$ are the reduced channels momenta.

Substitution (23) into boundary conditions (18) yields 
the following set of equations:

$$
\begin{gathered}
\sum_{n}\left\{\delta_{m n} e^{-i p_{n} x}\left(\alpha \partial_{x}+g A_{n}^{-}+i k_{n}\right)-S_{m n} e^{i p_{n} x}\right. \\
\left.\times\left(\alpha \partial_{x}+g A_{n}^{+}-i k_{n}\right)\left|\chi_{n}(x)\right\rangle\right\}=0,
\end{gathered}
$$

where $p_{n}=\alpha k_{n}$ are renormalized channels momenta and $g=\beta^{2} \sqrt{1+\alpha^{2}}$. To determine the operators $A_{n}{ }^{+}$one needs to calculate the action of the integral operator $Q(E$ $\left.+d^{2} / d y^{2}\right)$ on the elements $\psi_{n}^{ \pm}(x, y)=e^{ \pm k_{n} y} \chi_{n}(x)$ considered as the functions of the only variable $y=\alpha x$,

$$
\begin{aligned}
Q(E & \left.+\frac{d^{2}}{d y^{2}}\right) \psi_{n}^{ \pm}(y / \alpha, \gamma) \\
& =e^{ \pm i p_{n} x} Q\left(E+\left(\frac{1}{\alpha} \partial_{x} \pm i k_{n}\right)^{2}\right) \chi_{n}(x) \\
& =e^{ \pm i p_{n} x} \frac{1}{2 \pi i} \oint_{\Gamma} \Delta(\zeta) \varphi_{n}^{ \pm}(x, E-\zeta) d \zeta \\
& \equiv-e^{ \pm i p_{n} x} A_{n}^{ \pm}\left|\chi_{n}\right\rangle .
\end{aligned}
$$

Here the functions $\varphi_{n}^{ \pm}(x, \lambda)$ are the $L^{2}$ solutions of the boundary-value problem

$$
\left[(1 / \alpha) \partial_{x} \pm i k_{n}\right]^{2} \varphi_{n}^{ \pm}+\lambda \varphi_{n}^{ \pm}=\chi_{n},\left.\quad \varphi_{n}^{ \pm}\right|_{x=0}=0 .
$$

If the channel $n$ is opened, $\operatorname{Im} k_{n}=0$, then $\varphi_{n}^{+}=\overline{\varphi_{n}^{-}}$.

Formally, the $S$ matrix can be determined by projecting the system (24) on the basis $\left\{\chi_{n}\right\}$. In this case the system (24) can be written in terms of the matrices $W^{ \pm}$, playing the role of the wave operators

$$
W_{m n}^{ \pm}=\left\langle\chi_{m}\left|e^{ \pm i p_{n} x}\left(\alpha \partial_{x}+g A_{n}^{ \pm} \mp i k_{n}\right)\right| \chi_{n}\right\rangle
$$

in the following way:

$$
W^{+} S=W^{-} .
$$

On the opened channels we obviously have $\left(W_{m n}^{+}\right)^{*}=W_{m n}^{-}$.

\section{RESUME}

The next step is the studying of analytical properties of the suggested $S$ matrix

$$
S=\left(W^{+}\right)^{-1} W^{-} .
$$

It will be done anywhere. Let us note here the following circumstances.

(1) The scheme of the exclusion of the channels $\mathscr{H}^{b}$ and $\mathscr{H}^{q}$ described above demonstrates that the scattering on DQB can be reduced to some effective matrix-many-channel problem in the space $\mathscr{H}_{2}$,

$$
\left(-\frac{d^{2}}{d y^{2}} \otimes I+B-E\right) \Phi=0,
$$

with energy-dependent boundary conditions

$$
\left.\boldsymbol{\Phi}^{-1} \boldsymbol{\Phi}^{\prime}\right|_{\boldsymbol{y}=0}=\mathscr{P}(\boldsymbol{E}) \text {. }
$$

Here $B$ is a diagonal matrix of thresholds

$$
\begin{gathered}
B=\operatorname{diag}\left\{\epsilon_{n}\right\}, \\
P(E)=-i K^{1 / 2}\left(W^{+}+W^{-}\right)^{-1} \\
\left(W^{+}-W^{-}\right) K^{1 / 2}
\end{gathered}
$$

is a $P$ matrix ${ }^{7}$ of the problem,

$$
K=\operatorname{diag}\left\{k_{n}\right\} \text {. }
$$

(2) In the opened-channel approximation we can use the finite set of equations

$$
\sum_{n=1}^{N} W_{m n}^{+} S_{n l}=W_{m l}^{-}
$$

Denoting by $\Delta^{+}$the determinate of the matrix $W^{+}$,

$$
\Delta^{+}=\operatorname{det} W^{+}=\Delta^{+}\left(k_{1}, k_{2}, \ldots, k_{n}\right),
$$

we find

$$
S_{m l}=\left(1 / \Delta^{+}\right) \Delta^{+}\left(k_{1}, k_{2}, \ldots, k_{l-1}, k_{m}, k_{l+1}, \ldots, k_{n}\right) .
$$

Here we treat the channel momenta $k_{n}$ as independent variables and consider matrix element $S_{m l}$ as the function of these variables.

The studying of analytical properties of the $S$ matrix can be reduced to the investigation of corresponding Fredholm determinants $\Delta^{+}$(Ref. 8).

Nevertheless the most interesting question is to study the limit $N \rightarrow \infty$ and analytical behavior of the the total $S$ matrix.

In conclusion, we have demonstrated that the new method of coupling of different degrees of freedom in the system proposed, leads to a rather interesting scattering problem here. For more realistic simulation, the peripheral interaction in the Hamiltonian $\mathrm{H}_{2}$, as well as higher partial waves in the corresponding channel, should be taken into account.

\section{ACKNOWLEDGMENTS}

We wish to thank F. Lenz for fruitful discussions. One of the authors (K. A. M.) is indebted to the Fachbereich Physik for hospitality received during his stay at the Freie Universität Berlin where this work was finished.

'B. S. Pavlov, Teor. Mat. Fiz. 59, 346 (1984).

${ }^{2}$ Yu. A. Kuperin, K. A. Makarov, and B. S. Pavlov, Teor. Mat. Fiz. 63, 78 (1985); 69, 100 (1986).

${ }^{3}$ Yu. A. Kuperin, K. A. Makarov, and Yu. B. Mel'nikov, Teor. Mat. Fiz. 74, 103 (1988).

${ }^{4}$ Yu. A. Kuperin, K. A. Makarov, S. P. Merkuriev, B. S. Pavlov, and A. K. Motovilov, ITP Budapest Report No. 441, 1986.

${ }^{5}$ Y. Nogami and L. Tomio, Can. J. Phys. 62, 260 (1984); G. E. Brown, J. W. Durso, and M. B. Johnson, Nucl. Phys. A 397, 447 (1983).

'F. Lenz, J. T. Londergan, E. J. Moniz, R. Rosenfelder, M. Stingl, and K. Yazaki, Ann. Phys. 170, 65 (1986).

${ }^{7}$ I. M. Narodetskii, in Few-Body Problem in Physics, edited by L. D. Faddeev and T. I. Kapaleishvili (World Scientific, Singapore, 1985), Vol. 55. ${ }^{R}$ R. G. Newton, Scattering Theory of Waves and Particles (McGraw-Hill, New York, 1966). 